\title{
Excepcionalismo verde y desarrollo sostenible en Costa Rica
}

\author{
Green exceptionalism and sustainable development in Costa Rica
}

\author{
Alonso Ramírez Cover \\ Universidad de Costa Rica \\ San José, Costa Rica \\ alonso.ramirezcover@ucr.ac.cr
}

\begin{abstract}
RESUMEN. La sección temática del volumen 11 del Anuario del Centro de Investigación y Estudios Políticos tiene como propósito estudiar la compleja tensión entre la imagen de responsabilidad ambiental y armonía social que Costa Rica proyecta al proyecta al mundo y su conflictiva realidad a la hora de balancear entre sostenibilidad ambiental, justicia social y el desarrollo económico. Los artículos que componen esta sección exploran las múltiples dimensiones de esta confrontación y la forma en que esta se desenvuelve en una variedad de sectores, incluyendo el desarrollo agropecuario, la conservación ambiental, la producción energética, el turismo y las políticas sobre cambio climático. Sabemos bien que dilemas y conflictos similares suceden en otros países alrededor del mundo, pero Costa Rica es un caso especial. Aunque es un país pequeño y no muy importante en la geopolítica global, históricamente, ha asumido un rol crítico como sitio de experimentación de políticas para el desarrollo sostenible. Esto ha reforzado la idea de Costa Rica como un lugar "excepcional", previamente sustentada por la presencia de un sistema democrático progresivo, en una región caracterizada por el conflicto armado y el autoritarismo. Esta introducción ofrece un contexto económico-político de las formas peculiares en que Costa Rica ha sido construida discursivamente como un lugar excepcional a escala global.
\end{abstract}

PALABRAS CLAVE. Excepcionalismo verde, desarrollo sostenible, Costa Rica, neoliberalismo, imaginarios geopolíticos

ABSTRACT. The thematic section of the $11^{\text {th }}$ edition of the Anuario del 
Centro de Investigación y Estudios Políticos explores de complex tension between the imagen of environmental responsibility and social harmony that Costa Rica seeks to project to the world, and its conflictive reality of balancing sustainability, social justice and economic development. The articles that compose this section explore the multiple dimensions of this confrontation and the way it unfolds in a variety of sectors, including agricultural development, environmental conservation, energy production, tourism, and climate change policy. We now very well that similar conflicts and dilemmas are part of the reality of other countries around the world, but Costa Rica is a special case. Though it is a small and unimportant country in global geopolitics, it has historically assumed a critical role as a testing site for sustainable development policies. This has reinforced the idea of Costa Rica as an exceptional place, previously supported by the image of a progressive democratic system in a region characterized by armed conflict and authoritarianism. This introduction offers a political-economic context of the peculiar ways in which Costa Rica has been discursively constructed as an exception in global affairs.

KEYWORDS. Green exceptionalism, sustainable development, Costa Rica, neoliberalism, geopolitical imaginaries

\section{INTRODUCCIÓN}

El objetivo de la sección temática de este volumen es analizar la compleja tensión entre la imagen de responsabilidad ambiental y armonía social que Costa Rica proyecta al mundo y su conflictiva realidad a la hora de balancear los objetivos de sostenibilidad ambiental, justicia social y el desarrollo económico. Los artículos que componen esta sección exploran las múltiples dimensiones de esta confrontación y la forma en que esta se desenvuelve en una variedad de sectores, incluyendo el desarrollo agropecuario, la conservación ambiental, la producción energética, el turismo y las políticas sobre cambio climático. Se sabe bien que dilemas y conflictos similares suceden en otros países alrededor del mundo, pero Costa Rica es un caso especial. Aunque es un país pequeño y no muy importante en la geopolítica global, históricamente, ha asumido un rol crítico como sitio de experimentación de políticas para el desarrollo sostenible. Esto ha reforzado la idea de Costa Rica como un lugar "excepcional", previamente sustentada por la presencia de un sistema democrático progresivo, en una región caracterizada por el conflicto armado y el autoritarismo (Edelman, 1999).

Un aspecto común de los artículos de esta sección es que han 
sido desarrollados desde la óptica de la ecología política, un abordaje interdisciplinario que interpreta cómo el uso y la conservación de los recursos naturales está moldeado por la política (Robbins, 2013). Surgida originalmente como una subdisciplina que combinó el análisis de la ecología humana con el análisis marxista de los conflictos ambientales surgidos de desigualdades económico-políticas (Biersack, 2006), la ecología política se ha diversificado en las últimas tres décadas para incorporar abordajes posestructuralistas, principalmente basados en el trabajo de Michel Foucault (ver Agrawal, 2005; Fletcher, 2010) y otras perspectivas originadas en las ciencias sociales latinoamericanas (ver Gudynas, 2014). Aunque hay diferentes enfoques y tradiciones en la ecología política, se comparte un marco ontológico y epistemológico común basado en al menos tres principios. Primero, la ecología política parte de la suposición de que la sociedad desarrolla formas de organización y comportamiento, en parte, como resultado de sus esfuerzos para adaptarse a sus entornos naturales (Bryant, 1999). De este modo, segundo, la degradación ambiental a nivel local sólo puede entenderse en términos del desenvolvimiento de complejas fuerzas económico-políticas y culturales que a menudo trascienden dicha escala (Watts y Peet, 2004). En efecto, a diferencia de otras tendencias teóricas, la ecología política busca conocer cómo las decisiones locales sobre el uso de los recursos naturales están influenciadas por dinámicas que operan a escala nacional o global (Leff, 2003).

Tercero y ligado con lo anterior, una preocupación fundamental de la ecología política es el poder y, más específicamente, cómo las diferencias en el acceso al poder determinan los aspectos de la relación entre sociedad y naturaleza (Stott y Sullivan, 2000). En parte, esto tiene que ver con la manera en que los costos y los beneficios de las relaciones socioambientales son distribuidos - i.e.: quién se beneficia del control de ciertos recursos o paisajes socionaturales, quién sufre las consecuencias y quién tiene el poder de institucionalizar una visión del medioambiente que defienda esta distribución de beneficios (Ribot y Peluso, 2003). Múltiples estudios en ecología política demuestras cómo la desposesión del acceso a los recursos naturales son mediados por la imposición de discursos y suposiciones por parte de actores poderosos sobre qué es el medioambiente y cuál debería ser el rol humano relativo a éste (Castree y Braun, 1998).De este modo, en este marco analítico, el desarrollo sostenible es todo menos apolítico. Al contrario, está constituido por iniciati- 
vas fuertemente entrecruzadas por la proyección del poder político y las desigualdades sociales en diferentes escalas (Robbins, 2013).

A continuación, se abordarán las peculiares maneras en que Costa Rica ha sido construida discursivamente como un lugar excepcional a escala global, primero a partir de su pasado imaginado de democracia e igualdad, luego producto de su Estado de bienestar, y más tarde como una "república verde" (Evans, 1999). Se busca comprender cómo es que este país ha logrado convertirse en un modelo mundial para el desarrollo sostenible, y simultáneamente, un espacio para la degradación de los recursos naturales en favor del crecimiento económico. La respuesta descansa en la "excepcional" manera en que Costa Rica ha eludido algunos patrones comunes de la reestructuración neoliberal (ver Ong, 2006), lo cual tiene que ver con cómo el país y sus políticas de sostenibilidad han surgido a partir de la configuración particular de su economía política relativo a otras fuerzas globales.

\section{EXCEPCIONALISMO COSTARRICENSE}

El seductor pasado imaginado de Costa Rica como una nación excepcional es una construcción histórica cuyos orígenes son fuertemente debatidos hoy. Temprano en la historia nacional se postuló que la pobreza colonial del país relativa a otras partes de Centroamérica impidió la formación de las economías de plantaciones que caracterizaron el istmo. Consecuentemente, Costa Rica alcanzó su independencia como una nación de pequeños propietarios agrícolas, cosa que permitió la implementación de una democracia igualitaria (Seligson, 2001a). Como evidencia, se ha señalado que el país no experimentó períodos tan largos de dictadura militar o guerra civil desde su independencia, diferente de otras naciones centroamericanas (Gudmundson, 1990; Gutiérrez Arguedas y Granados Chaverri, 2020).

No obstante, estudios contemporáneos cuestionan este relato, argumentando que, en realidad, el período inmediatamente posterior a la independencia fue marcado por la conflictividad social y la disputa política provocada por la competencia entre las élites por el control del estado (Acuña Ortega, 2002). La inestabilidad fue superada con la consolidación de un liderazgo militar autoritario durante las décadas de 1870 y 1880, que permitió consolidar la autoridad política a favor de élites políticas favorables a la expansión 
de la agroexportación capitalista, cuyo propósito fue brindar progreso liberal mediante la "civilización" de las clases más bajas de la población (Molina, 1988). Precisamente, consideraciones como éstas motivaron la formación de un sistema público de educación destinado a reducir el analfabetismo y consolidar una cultura política nacional. En medio de la formación de este Estado liberal, una elección controversial en 1889 derivó en un alzamiento popular que es frecuentemente invocado como el origen de la democracia costarricense (Mahoney, 2003). Sin embargo, debe recordarse que las intrigas entre las élites económicas prosiguieron durante la primera mitad del siglo XX, a pesar de que las transferencias de poder fueron regulares (Edelman, 1999).

Para estos autores, el origen del excepcionalismo costarricense es el modelo de gobernanza social demócrata que fue emergiendo gradualmente durante el siglo XX, en contradicción con el Estado liberal (Seligson, 2001b), consolidándose con un fuerte estado de bienestar en la década de 1940. Esto fue el resultado de una coalición inusual de algunos intereses agroexportadores, la Iglesia Católica y el Partido Comunista que habían iniciado una serie de ambiciosas reformas sociales a nivel estatal, para atender los efectos económicos globales de la Depresión de los 1930 y la Segunda Guerra Mundial en Costa Rica (Edelman, 1999). Aunque las tensiones de fondo dieron cabida a la Guerra Civil en 1948 - el episodio de violencia política más serio de la historia nacional (Molina y Palmer, 2017) el resultado fue una reforma institucional profunda que mantuvo las incipientes reformas sociales y las expandió en la forma de un Estado de Bienestar con mayores poderes de intervención en la economía nacional, al tiempo que suprimió y cooptó a las fuerzas más radicales de izquierda (Rovira, 1987).

El período de posguerra promovió una expansión de este Estado de Bienestar, facilitada por una de las más altas tasas de crecimiento económico en Latinoamérica durante las décadas de 1950 y 1960 (Edelman, 1999). Este crecimiento se debía a las demandas de una economía global que se expandía exponencialmente y que se centralizaba en Estados Unidos como principal mercado de exportación global, así como en el fortalecimiento de la relación geopolítica entre Costa Rica y ese país. En efecto, el gobierno estadounidense interpretaba a Costa Rica como un importante aliado latinoamericano contra el comunismo, sobre todo después de la Revolución Cubana de 1959 (Booth et al., 2010). Esta relación se vio manifesta- 
da en un influjo significativo de inversión extranjera directa y ayuda internacional al desarrollo la cual alcanzó los 4 mil millones de dólares entre 1940 y 1960 (Aistara, 2018). Precisamente, esta interacción dio solidez a una imagen de Costa Rica en la cooperación internacional como un país políticamente estable y muy pacífico, pero así también muy receptivo a las ideas e influencia externa. Todo esto empieza a dar forma a un código geopolítico nacional que busca dar certeza de Costa Rica como un país con el que es "muy fácil trabajar", en términos de experimentación de nuevas políticas surgidas de la cooperación internacional.

En la década de 1970, el modelo estatista fue ampliado para contener la recesión global, intensificando el control del Estado sobre diferentes sectores productivos con la creación de nuevas empresas públicas y entidades autónomas (Rovira, 1987). Así, para la década siguiente, el Estado ejercía una influencia considerable en la sociedad, empleando a un quinto de la fuerza de trabajo y controlando un cuarto del producto interno bruto (Edelman, 1998), al tiempo que las políticas sociales habían logrado estándares de vida semejantes a la mayoría de los países en vías de desarrollo (Evans, 1999). Así las cosas, la combinación de un estado de bienestar basado en un modelo reformista y conciliatorio en combinación con un sistema electoral estable (aunque excluyente de opciones políticas radicales) fueron contempladas como las bases del excepcionalismo costarricense.

\section{EXCEPCIONALISMO VERDE}

Desde la década de 1990, Costa Rica ha venido proyectándose como una eco-utopía en el imaginario global, llevando al surgimiento de epítetos sobre el país como una "república verde" (Evans, 1999) y dando cabida a una vertiente en la narrativa nacional como una excepción global. Sin embargo, esta no ha sido siempre la imagen ambiental del país. Históricamente, la economía costarricense ha estado principalmente basada en la extracción de recursos naturales para su procesamiento y venta en mercados internacionales, incluso antes de la producción del café (Gudmundson, 1990). El café vino a consolidar esta articulación periférica del país, que luego se consolidó con el banano en plantaciones, y posteriormente con otras mercancías agropecuarias como la caña de azúcar, la ganadería, la palma aceitera y, más recientemente, la piña (Edelman, 1999). El 
modelo de crecimiento basado en la agroexportación tuvo efectos ambientales nocivos (Herrera, 2013). Precisamente, en el contexto del Estado de Bienestar, la implementación de nuevos incentivos legales para la conversión de bosques en plantaciones y pastizales ganaderos produjeron una rápida expansión de la frontera agropecuaria (Brockett y Gottfried, 2002). Para la década de 1970, la articulación de la agroindustria ganadera costarricense con la cadena global de la carne y la comida rápida había provocado una marcada deforestación (Edelman, 1998). Así las cosas, en treinta años, la cobertura forestal había pasado del 50\% del territorio nacional a menos del 20\% (Evans, 1999).

Esta degradación forestal se convirtió en un problema político, inspirando campañas para proteger los bosques nacionales que habían sobrevivido la expansión agrícola (Campbell, 2002). Esto derivó en la promulgación de la Ley Forestal de 1969, la cual creó un Departamento de Parques Nacionales responsable de expropiar parcelas de bosque en la frontera agrícola para ponerlas bajo protección estatal, con la intención de preservarlas de la expansión agropecuaria que otras agencias estatales promovían agresivamente mediante incentivos económicos y legales (Sader y Joyce, 1988). Allí comienza la imagen contemporánea de Costa Rica como república verde, la cual termina consolidándose durante los 1990 debido al desarrollo de un sistema extenso de áreas protegidas y la promoción del ecoturismo como una forma crucial de generación de divisas internacionales e ingresos basado en estos esfuerzos de conservación. En el núcleo de este imaginario se encuentran los parques nacionales, que, en conjunto con otros tipos de áreas protegidas, cubren más de un cuarto del territorio nacional y cuentan con un amplio reconocimiento internacional. Precisamente, Costa Rica cuenta con una de las tasas de protección territorial más altas del mundo, excediendo la ambiciosa meta del $17 \%$ global establecida por las Metas de Aichi de la Convención de Diversidad Biológica (Brockington et al., 2008), aspecto que ha derivado en el reconocimiento del país como una "superpotencia de la conservación" (Boza et al., 1995).

El modelo de conservación empleado en Costa Rica fue consistente con la naturaleza de la gobernanza estatista y las tendencias dominantes en la cooperación internacional favorable al preservacionismo que imperaba en la década de 1970. Así, se terminó optando por la creación de áreas protegidas socialmente excluyentes 
y establecidas mediante la expropiación de tierras y la evicción sistemática de sus antiguos habitantes, en lo que Dan Brockington llama "modelo de fortaleza" (Brockington, 2002). El proceso fue (y sigue siendo) apoyado financiera y técnicamente por donantes internacionales (Isla, 2015). Originalmente, estas facetas hicieron a las áreas protegidas impopulares entre el campesinado y otros sectores del país y su supervivencia resultó garantizada una vez que la conservación fue asociada a su capacidad de generar ingresos, lo cual motivó a explorar el potencial de estas áreas para promover el ecoturismo. Durante los 1990, Costa Rica logró consolidar una reputación internacional como destino de turismo basado en la naturaleza (Honey, 1999), la cual fue reforzada mediante el uso de exitosas campañas publicitarias del Instituto Costarricense de Turismo (ICT) que afirmaban un país "sin ingredientes artificiales". Para inicios de los 1990, el turismo producía más divisas que el café y el banano, alcanzando un 8\% del PIB (Honey, 1999, pp. 163-164). Durante 2017, Costa Rica recibió una cantidad de turistas equivalente a más de la mitad de su población, con un $65 \%$ de éstos citando el disfrute del ecoturismo como su principal razón de visita del país (ICT, 2017). Rápidamente, el país comenzó a recibir una importante inversión extranjera orientada al turismo y los bienes raíces (Barrantes Reynolds, 2011) dando cabida a hoteles "ecológicos" grandes y pequeños para atender segmentos de alto precio, con muchos de sus dueños convirtiéndose en consultores internacionales del ecoturismo mundial (Fletcher, 2014).

En fechas recientes, la imagen "verde" de Costa Rica ha sido reforzada por esfuerzos nacionales para el combate del cambio climático. Basándose en el hecho de que las empresas públicas y privadas que generan energía eléctrica utilizan fuentes no fósiles, como represas hidroeléctricas, plantas eólicas y geotérmicas (Zárate Montero y Ramírez García, 2016), el país ha sido planteado como un modelo de energías "limpias". Esto también ha reforzado la atención internacional sobre la meta asumida por la segunda administración de Oscar Arias en 2007 de convertir a Costa Rica en el primer país carbono neutro del mundo para el 2021. Diferentes organizaciones ambientalistas han identificado al país como un modelo a seguir, citando como evidencia estas políticas, el hecho de que Costa Rica fue el primer país en introducir el pago por servicios ambientales para financiar la conservación forestal y su rol como promotor de REDD+ para reducir la deforestación y la 
degradación forestal usando mercados de carbono (Matulis, 2013).

No obstante, la realidad social y ambiental se alejada de la imagen internacional que se proyecta. Simultáneo a este "boom de la conservación", desde los 1980, Costa Rica también experimentó aumento en el área dedicada para agricultura industrial en la forma de plantaciones de piña, palma aceitera, banano y ganadería (Zimmerer, 2011). Esta producción ha resultado ambientalmente destructiva por su expansión territorial, pero también por el uso intensivo de agroquímicos nocivos. De hecho, Costa Rica es uno de los países que utiliza mayores concentraciones de pesticidas per cápita en el mundo (Galt, 2014). Paralelamente, el país también enfrenta una crisis hídrica en sus cuencas más importantes, producto del poco tratamiento dado a las aguas residuales, la sobreutilización de fuentes de agua y las altas concentraciones de la contaminación en manantiales y ríos (ver: Alpízar, 2014; MINAE et al., 2013). Las ciudades costarricense crecen de forma insostenible y sin muchos controles sobre el uso racional de recursos naturales circundantes y la privatización de los espacios públicos (Montes Ruiz y Durán Segura, 2019). Aunque el país se contempla como modelo para la "energía limpia", los y las costarricense producen más toneladas de dióxido de carbono (CO2) per cápita que cualquier otro país centroamericano, a pesar de tener una población más pequeña (PEN, 2018). De modo que, el "excepcionalismo verde costarricense" está construido a partir de notables contradicciones.

Como en otros países, estas contradicciones resultan de las múltiples presiones nacionales e internacionales que acompañan las metas (en competencia) de desarrollo económico y protección ambiental en el sistema capitalista. Quizás lo que define a Costa Rica respecto a otros casos es, primero, la separación geográfica que evidencian estos dos procesos y que se refleja en la promoción de formas de conservación absoluta en algunas áreas con el desarrollo de plantaciones y agroindustria intensiva en otras. Segundo, destaca el rol contradictorio de las políticas estatales que promueven la producción en plantación y otras formas de extractivismo a través de diferentes incentivos (Vandermeer y Perfecto, 2013), pero que simultáneamente financia áreas protegidas y pagos por servicios ambientales (ver Obando en este volumen para un análisis de las contradicciones del rol del Estado en el caso de la producción piñera y las políticas de protección ambiental). El resultado es una competencia frenética entre la expansión productiva y la preser- 
vación socialmente excluyente, cada una auspiciada por su propia institucionalidad, cada una vinculada a formas diferentes de generación de divisas, y cada una buscando reclamar toda la tierra posible antes que el otro bando (Fletcher et al., 2020).

\section{¿EXCEPCIÓN AL NEOLIBERALISMO?}

Estas tensiones peculiares entre la conservación y el desarrollo se originan en la experiencia costarricense en la Crisis de la Deuda de la década de 1980. La prosperidad previamente descrita durante las décadas de 1960 y 1970 conllevó un importante costo en la forma de un aumento dramático de la deuda pública, la cual se cuadruplicó entre 1977 y 1981 (Rovira, 1987). Además, opuesto a quienes contemplan al pasado como mejores tiempos, los frutos de esta prosperidad nunca se distribuyeron equitativamente para el campesinado y el medioambiente (Edelman, 1999). Como señala Guillén (en este volumen), una historia que se repite en las zonas rurales costarricense es la de pequeños productores agropecuarios que buscaron organizarse y adoptar otras técnicas que les permitiera extraer algún beneficio, pero que resultaron frecuentemente sufriendo de diferentes formas de desplazamiento producto de la agricultura capitalista de gran tamaño y luego condenados a la pobreza, la expropiación, el éxodo y la ocupación ilegal. A finales de los 1970, el aumento del precio del petróleo, la caída en los precios del café y el crecimiento de las tasas de interés sobre la deuda pública, provocaron que Costa Rica declarara una moratoria en el pago de sus obligaciones en 1981, y registrar una de las deudas per cápita más altas del mundo (Hidalgo Capitán, 2003).

Costa Rica se vio obligada a administrar una crisis tripartita en los 1980. La crisis macroeconómica y la atención de la deuda externa obligó al país a asumir tres rondas de ajuste estructural bajo supervisión del Banco Mundial y el Fondo Monetario Internacional en 1985, 1988 y 1993 (Rovira, 1987). Estos procesos demandaron una reducción significativa del gasto público, la privatización de una parte del sector público, y la eliminación de aranceles a las importaciones que protegían a la economía doméstica de la competencia internacional. Simultáneamente, el ajuste orientó la economía nacional a las exportaciones no tradicionales, incluyendo la producción textil y la exportación de piña y otras mercancías para aumentar la competitividad internacional (Hidalgo Capitán, 
2003). También se establecieron beneficios fiscales para promover la inversión extranjera directa y la eliminación de apoyo a precios para cultivos de consumo nacional como el maíz, el arroz y los frijoles. Estas reformas pretendieron redirigir el desarrollo nacional hacia fuera.

Simultáneo con estas reformas, el país también se encontraba bajo una presión considerable de los Estados Unidos para que abandonara su posición de neutralidad respecto a la Guerra Civil en Nicaragua, bajo la rúbrica de que el territorio nacional se usara como base militar para los contrarrevolucionarios nicaragüenses (Honey, 1999). En efecto la administración estadounidense de ese momento veía a Costa Rica como una potencial vitrina de una democracia capitalista próspera y estable, frente a las dictaduras militares de Centroamérica. Dada su súbita significancia geopolítica, Costa Rica recibió grandes transfusiones de ayuda internacional, permitiéndole atender los efectos más negativos de la recesión económica (Edelman, 1999). Esto dio una influencia política considerable a la Agencia de Desarrollo de Estados Unidos (USAID) y al Departamento de Estado en los asuntos públicos nacionales (ver Robinson, 2003; Sojo, 1991).

Pero, a medida que el ajuste se desarrollaba, Costa Rica también experimentaba una seria crisis ambiental. Desde la década de 1960, el país experimentaba una de las tasas de deforestación más altas del mundo, resultado de los incentivos desarrollistas a la producción agropecuaria y luego, el apoyo neoliberal a nuevas formas de agricultura de exportación (Sader y Joyce, 1988). Inicialmente un problema no atendido por el ajuste estructural, la deforestación en Costa Rica se convertiría en un asunto de interés para los organismos financieros internacionales hacia finales de la década de 1980 (USAID, 1989), por considerar la futura dependencia nacional a la importación de madera como una amenaza futura a la balanza comercial (Lansing et al., 2015). Una multitud de políticas serían establecidas para promover un cambio orientado hacia una gobernanza ambiental neoliberal, dirigida a la optimización del mercado para la gestión y protección de los bosques y la biodiversidad (Ramírez, 2017). En pocas palabras, estas tres diferentes crisis - de deuda, geopolítica y ambiental - y las formas de presión y asistencia que fueron subsecuentemente implementadas en el país, establecieron una coyuntura clave para el afianzamiento del neoliberalismo, las lógicas de mercado y la estandarización de 
nuevos métodos para calcular, clasificar y organizar los recursos naturales de la nación. Estas nuevas "racionalidades" son fácilmente observables en el desarrollo institucional costarricense relativo a la protección del medioambiente y la promoción de la agricultura, el turismo, la producción energética y la descarbonización (ver Aistara, 2018; Fletcher y Breitling, 2012; Galt, 2008; Kuzdas et al., 2015; Lansing, 2011; Meletis y Harrison, 2010; van Noorloos, 2011; Zimmerer, 2011).

En el tema ambiental, la neoliberalización ha tenido importantes consecuencias. Entre 1980 y 1990, el personal del Servicio de Parques Nacionales costarricense fue reducido en un $20 \%$ y la inversión en equipo y mantenimiento cayó en más de un $80 \%$ (Steinberg, 2001). La reducción de los fondos para el manejo de parques se convirtió en un componente clave del ajuste estructural, llevando a una "brecha de gobernanza" que fue pronto llenada por los organismos financieros y las organizaciones no gubernamentales, fuertemente apoyadas por nuevas dinámicas de financiamiento basadas en cambios de deuda por naturaleza y otros fondos privados. En efecto, estas organizaciones se volvieron sumamente influyentes en los esfuerzos de conservación, dada la retirada del gobierno costarricense. Esto dio cabida a una suerte de división del trabajo en la conservación, con el Estado costarricense asumiendo la protección de las áreas protegidas en modalidad de fortaleza, y las ONG y otras organizaciones tomando responsabilidad por el trabajo con las comunidades en o alrededor de estas áreas.

La secuela más notable del ajuste estructural, dadas estas condiciones financieras, geopolíticas y ambientales, es que Costa Rica, irónicamente, ha terminado en una posición privilegiada como uno de los "laboratorios" de avanzada global para la prueba y experimentación con nuevas políticas ambientales y para la promoción de actividades intensivas en el uso de recursos naturales. Desde la implementación de nuevas categorías de manejo para áreas protegidas, al ecoturismo, los programas de pago por servicios ambientales y las recientes estrategias de descarbonización, el país ha logrado posicionarse exitosamente a la vanguardia de la gestión sostenible de recursos y la conservación ambiental (Boza et al., 1995). Pero, a la vez, a través de nuevas técnicas de injertado y manipulación genética de cultivos, la aplicación de agentes químicos para elevar la productividad, experimentos en la relocalización de gentes, plantas, semillas y sustancias y el desarrollo de investiga- 
ciones de capital para promover la homogeneización y control de espacios rurales, y la promoción de formas de descarbonización amigables con el uso intensivo de recursos, Costa Rica también se ha convertido en punta de lanza para la experimentación con economías de plantación (ver Edelman, 1999; Espinasa et al., 2017; Flagg, 2015; Galt, 2014; Goldstein, 2001; Segura y Moreira, 2013). La paradoja de todo esto es que el país ha surgido contradictoriamente como un centro global para la conservación de la naturaleza y como un epicentro de la modernización productiva basada en el uso intensivo de recursos naturales mediante la agroexportación de monocultivos, el turismo y la producción energética.

Entre 1985 y 2019, la cantidad de áreas protegidas en Costa Rica se triplicó, mientras que el área total bajo protección aumentó en más del 70\% (ver Zimmerer, 2011; SINAC, 2009). Para lograr esto, mecanismos y capacidades legales, institucionales y administrativas que habían sido establecidos previamente ${ }^{1}$ fueron reorganizados a través de las políticas neoliberales del ajuste, promoviendo un "boom" en la conservación costarricense (Zimmerer, 2011). El Plan de Canje de Deuda por Naturaleza de 1987 constituyó un importante esfuerzo para financializar la agenda de conservación, estableciendo una línea de crédito fundamental para la compra de tierras para crear nuevas áreas protegidas y expandir las ya existentes (Evans, 1999). En 1998, la Ley de Biodiversidad (surgida del último plan de ajuste estructural) reemplaza al Departamento de Parques Nacionales con el Sistema Nacional de Áreas de Conservación (SINAC), un órgano descentralizado destinado a administrar las áreas protegidas mediante la incorporación de abordajes de conservación "fuera de sus fronteras", y el uso de nuevas categorías menos estrictas de protección, con el objetivo de promover el uso económico de la naturaleza bajo conservación, por medio del turismo y la bioprospección (Brockett y Gottfried, 2002). Finalmente, en 1996, se institucionaliza el programa de pago por servicios ambientales y se crea el Fondo Nacional de Financiamiento Forestal (FONAFIFO), implicando una aparente transición hacia el uso de

1 Antes de 1985, Costa Rica había logrado aprobar una serie de leyes importantes para promover la protección forestal (4465), la creación de parques nacionales (6084) y la creación y expansión de reservas biológicas (6794) en 1969, 1977 y 1982 , respectivamente. No obstante, estimaciones del área protegida total sugieren que el país fue poco exitoso en la creación de muchas áreas protegidas entre los 1970 y los 1980 (Brockett y Gottfried, 2002; Evans, 1999). 
instrumentos de mercado para la conservación (Fletcher y Breitling, 2012).

Simultáneo a esta expansión de las áreas protegidas, y bajo el auspicio de las políticas neoliberales, Costa Rica también experimentó una masiva modernización de su agricultura. Producto de una estrategia de desarrollo agrícola basada en la inserción a los mercados globales, la privatización de la tierra, los insumos productivos y la investigación y extensión agropecuaria, la producción de monocultivos no tradicionales para la exportación presentó crecimientos dramáticos (Galt, 2008). A inicios de los 1980, la palma aceitera y la piña tenían 14.000 hectáreas cultivadas y hoy se cultivan en más de 110.000. Aparte de una pequeña producción orgánica certificada y orientada a segmentos de 'fair trade' que ha logrado algunos pocos resultados ambientalmente sostenibles (Aistara, 2018), la mayoría de la producción agrícola para la exportación ha terminado contribuyendo con la destrucción ambiental. Análisis detallados de la zona norte del país demuestran que la expansión del cultivo de piña desde 1996 ha implicado reemplazos de pastizales y bosques (Fagan et al., 2013). Al igual que el banano y otros monocultivos de exportación, la piña es fuertemente producida con el uso de agroquímicos, recibiendo hasta 20 kilogramos de ingredientes activos por hectárea por ciclo productivo, lo cual ha resultado en serios impactos sociales y ambientales (ver Echeverría-Saénz et al., 2012; Lawrence, 2010). La expansión de monocultivos ha sido en todo momento apoyada por el Estado costarricense en conjunto con proyectos de inversión de empresas agropecuarias privadas y con el apoyo de organismos financieros internacionales (Segura y Moreira, 2013). Esta sección temática sigue la tradición de enmarcar a Costa Rica como un laboratorio internacional, elevando preguntas sobre qué podemos aprender de las políticas para incentivar el desarrollo económico y la conservación ambiental durante los últimos cuarenta años.

\section{CONTRIBUCIONES DE ESTA SECCIÓN TEMÁTICA}

Las obras que componen la sección temática de este volumen del Anuario del Centro de Investigación y Estudios Políticos ofrecen una amplia gama de abordajes constituyentes de la ecología política contemporánea y ofrecen hallazgos de diferentes sectores como agricultura, conservación ambiental, ecoturismo, política energé- 
tica y cambio climático, aunque apuntando a diferentes temas señalados en la introducción. Se comienza con el trabajo de Alexa Obando, el cual reflexiona sobre el contradictorio rol del Estado a la hora de balancear entre el crecimiento económico y protección ambiental. Explicando cómo el Estado promociona la inversión en el monocultivo de la piña, mientras que sus autoridades ambientales evitan involucrarse en disputas con este sector, la autora reflexiona sobre en la necesidad de analizar esta aparente falta de armonía a partir del uso de omisiones deliberadas para dar continuidad a la acumulación y el despojo.

Los artículos de María José Guillén, Tania Rodríguez y Marylaura Acuña se concentran en explorar otra faceta del excepcionalismo verde, el cual tiene que ver con cómo el desarrollo sostenible se implementa en zonas rurales bajo la rúbrica de intervenciones centradas en optimizar prácticas sociales, económicas y ambientales locales, mediante el mercado. En el caso de Guillén, su artículo ofrece una lectura muy convincente de cómo los nuevos abordajes del Instituto de Desarrollo Rural (INDER) implementados a la luz de la reforma neoliberal, derivan en programas de otorgamiento de tierras que, al establecer nuevos mecanismos disciplinarios centrados en la administración de deudas y cuotas de producción, pretenden construir una subjetividad empresarial entre los campesinos beneficiarios. Por su parte, Acuña estudia cómo estas mismas políticas para promover un mayor acceso a la tierra han sido ampliadas por el desarrollo sostenible para integrar mujeres en zonas rurales y resolver la brecha de género en el acceso a la tierra. Pero, contrario a esta visibilización del rol de las mujeres en la agricultura, la reforma neoliberal ha desmantelado el sector, marginalizando la participación de las mujeres en la producción.

Rodríguez, estudiando las estrategias utilizadas por organismos internacionales durante varias décadas de intervenciones para el fomento del cacao en Talamanca, concluye que saberes locales han sido estratégicamente apropiados con la intención de conceptualizar modelos de producción cacaotera que luego son diseminados entre las poblaciones indígenas para facilitar la adopción de culturas agropecuarias occidentales y articular estas poblaciones al mercado. Así las cosas, aunque las intervenciones estudiadas por María José Guillén, Marylaura Acuña y Tania Rodríguez pretendían "diversificar" la producción en estas zonas rurales de diferentes formas, está claro que estas "nuevas alternativas" dependen de ra- 
cionalidades limitadas a la maximización del valor económico y las prácticas de mercado. De este modo, este desarrollo sostenible a la tica (ver Monge, 2015) pareciera manifestarse en una "monocultura de la mente" (Shiva, 1993), i.e.: un proyecto para la estandarización del paisaje socioecológico con miras a garantizar la extracción de la riqueza económica y promover el control político de quienes lo habitan.

Uno de los propósitos fundamentales de esta sección temática es cuestionar los orígenes y las formas en que Costa Rica es imaginada como un sitio excepcional, al tiempo que se invisibilizan importantes contradicciones socioambientales. Al respecto, los trabajos de Santiago Navarro y Alberto Gutiérrez ofrecen excelentes análisis críticos desde el turismo y la generación de energía eléctrica, respectivamente. Mediante un estudio de las estrategias publicitarias empleadas por el Instituto Costarricense de Turismo, Navarro explica que el fantástico imaginario de excepcionalidad costarricense no depende exclusivamente de actores internacionales, sino que del trabajo de instituciones nacionales también. Navarro concluye que estas campañas refuerzan una suerte de "autotropicalismo", es decir, una forma de colonialismo interno en el que élites económicas locales no ven problemas en reproducir imaginaciones coloniales bien conocidas de los trópicos como lugares exóticos, pero domesticados, con la intención de motivar la visitación de turistas extranjeros. Mientras tanto, en el proceso, se invisibilizan los nocivos efectos sociales, laborales, políticos y ecológicos del turismo en las comunidades receptoras.

Por su parte, Gutiérrez ofrece un análisis crítico de cómo Costa Rica ha empezado a ser imaginada como un caso paradigmático en el desarrollo de energías limpias y un laboratorio mundial de la descarbonización, producto del uso mayoritario de fuentes hidroeléctricas en su matriz energética. Habiendo descartado las contradicciones obvias - i.e.: que se cuenta con una flota vehicular de notables proporciones que funciona con combustibles fósiles, que el país cuenta con una de las huellas de carbono más grandes de Centroamérica y que la realidad de la construcción de represas hidroeléctricas es todo menos que sostenible - Gutiérrez explica cómo estas imaginaciones surgen de la intersección de intereses geopolíticos internos y externos. Internamente, se busca legitimar la hidroelectricidad en un contexto de tensiones relacionadas con la complejidad del modelo neoliberal de producción de energía pri- 
vada y recientes conflictos con otros usuarios de cuencas; y externamente, producto del reposicionamiento de la agenda de cambio climático hacia la mitigación de emisiones de carbono.

\section{FUENTES CONSULTADAS}

Acuña Ortega, V. H. (2002). La invención de la diferencia costarricense, 1810-1870. Revista Historia, 45(1), 191-228.

Agrawal, A. (2005). Environmentality: Techonologies of government and the making of subjects. Duke University Press.

Aistara, G. A. (2018). Organic sovereignties: Struggles over farming in an age of free trade. University of Washington Press.

Alpízar, F. (2014). Poder y participación política en la gestión del agua en Costa Rica. Editorial Arlekín.

Barrantes Reynolds, M. P. (2011). The expansion of "real estate tourism" in coastal areas: Its behaviour and implications. Recreation in Society in Africa, Asia and Latin America, 2(1), 51-70.

Biersack, A. (2006). Reimagining political ecology: Culture/power/history/nature. En A. Biersack y J. B. Greenberg (Eds.), Reimagining political ecology. Duke University Press.

Booth, J. A., Wade, C. J., y Walker, T. W. (2010). Understanding Central America: Global forces, rebellion, and change. Westview Press.

Boza, M. A., Jukofsky, D., y Wille, C. (1995). Costa Rica Is a Laboratory, Not Ecotopia. Conservation Biology, 9(3), 684-685. https://doi.or$\mathrm{g} / 10.1046 / \mathrm{j} .1523-1739.1995 .09030684 . x$

Brockett, C. D., y Gottfried, R. R. (2002). State policies and the preservation of forest cover: Lessons from contrasting public-policy regimes in Costa Rica. Latin American Research Review, 37(1), 7-40.

Brockington, D. (2002). Fortress conservation: The preservation of the Mkomazi Game Reserve, Tanzania. Indiana University Press.

Brockington, D., Duffy, R., y Igoe, J. (2008). Nature unbound: Conservation, capitalism and the future of protected areas. Earthscan.

Bryant, R. L. (1999). A political ecology for developing countries. Zeitschrift fur Wirtschaftgeographie, 43(3-4), 148-157.

Campbell, L. M. (2002). Conservation Narratives in Costa Rica: Conflict and Co-existence. Development and Change, 33(1), 29-56. https://doi. org/10.1111/1467-7660.00239

Castree, N., y Braun, B. (1998). The construction of nature and the nature 
of construction. Remaking reality: Nature at the millenium, 3-42.

Echeverría-Saénz, S., Mena, F., Pinnock, M., Ruepert, C., Solano, K., de la Cruz, E., Campos, B., Sánchez-Avila, J., Lacorte, S., y Barata, C. (2012). Environmental hazards of pesticides from pineapple crop production in the Río Jimenez watershed (Caribbean coast, Costa Rica). Science of the Total Environment, 440, 106-114.

Edelman, M. (1998). La lógica del latifundio: Las grandes propiedades del noroeste de Costa Rica desde finales del siglo XIX. Editorial UCR.

Edelman, M. (1999). Peasants Against Globalization: Rural Social Movements in Costa Rica. Stanford University Press.

Espinasa, R., Balza, L., Hinestrosa, C., Sucre, C., y Anaya, F. (2017). Dossier energético: Costa Rica. Banco Interamericano de Desarrollo. https:/ / doi.org/10.18235/0000588

Evans, S. (1999). The Green Republic: A conservation history of Costa Rica. University of Texas Press.

Fagan, M. E., de Fries, R. S., Sesnie, S. E., Arroyo, J. P., Walker, W., Soto, C., Chazdon, R. L., y Sanchun, A. (2013). Land cover dynamics following a deforestation ban in northern Costa Rica. Environmental Research Letters, 8(3).

Flagg, J. (2015). Aiming for zero: What makes nations adopt carbon neutral pledges. Environmental Sociology, 1(3), 202-212.

Fletcher, R. (2010). Neoliberal environmentality: Towards a poststructuralist political ecology of the conservation debate. Conservation and Society, 8(3), 171-181.

Fletcher, R. (2014). Romancing the wild: Cultural dimensions of ecotourism. Duke University Press.

Fletcher, R., y Breitling, J. (2012). Market mechanism or subsidy in disguise? Governing payments for environmental services in Costa Rica. Geoforum, 43(3), 402-411.

Fletcher, R., Dowd-Uribe, B., y Aistara, G. A. (Eds.). (2020). The ecolaboratory: Environmental governance and economic development in Costa Rica. University of Arizona Press.

Galt, R.E. (2014). Food systems in an unequal world: Pesticides, vegetables and agrarian capitalism in Costa Rica. University of Arizona Press.

Galt, R.E. (2008). Pesticides in export and domestic agriculture: Reconsidering market orientation and pesticide use in Costa Rica. Geoforum, 39(3), 1378-1392. https://doi.org/10.1016/j.geoforum.2007.12.003

Goldstein, D. (2001). Financial sector reform and sustainable develop- 
ment: The case of Costa Rica. Ecological Economics, 37(2), 199-215. https://doi.org/10.1016/S0921-8009(00)00278-0

Gudmundson, L. (1990). Costa Rica antes del café: Sociedad y economía en vísperas del boom agroexportador. Editorial UNED.

Gudynas, E. (2014). Ecologías políticas: Ideas preliminares sobre concepciones, tendencias, renovaciones y opciones latinoamericanas. Centro Latinoamericano de Ecología Social.

Gutiérrez Arguedas, A., y Granados Chaverri, A. (2020). Nacionalismo, frontera y excepcionalismo verde en Costa Rica. Anuario de Estudios Centroamericanos, 46(1), 1-28.

Herrera, M. (2013). Sustainable development in Costa Rica: A geographic critique. Journal of Latin American Geography, 12(3), 193-219.

Hidalgo Capitán, A. L. (2003). Costa Rica en evolución: Política económica, desarrollo y cambio estructural del sistema socioeconómico costarricense (1980-2002). Editorial UCR y Servicio de Publicaciones de Universidad de Huelva.

Honey, M. (1999). Ecotourism and sustainable development: Who owns paradise? Island Press.

ICT. (2017). Anuario Estadístico de Turismo 2017. Instituto Costarricense de Turismo.

Isla, A. (2015). The "greening" of Costa Rica: Women, peasants, indigenous peoples and the remaking of nature. Toronto University Press.

Kuzdas, C., Wiek, A., Warner, B., Vignola, R., y Morataya, R. (2015). Integrated and participatory analysis of water governance regimes: The case of the Costa Rican dry tropics. World Development, 66, 254-268.

Lansing, D., Grove, K., y Rice, J. (2015). The neutral state: Genealogy of ecosystems services payments in Costa Rica. Conservation and Society, 13(2), 200-211.

Lansing, D. M. (2011). Realizing Carbon's Value: Discourse and Calculation in the Production of Carbon Forestry Offsets in Costa Rica. Antipode, 43(3), 731-753. https://doi.org/10.1111/j.1467-8330.2011.00886.x

Lawrence, F. (2010). Bitter fruit: The truth about supermarket pineapple. The Guardian.

Leff, E. (2003). La ecología política en América Latina. Un campo en construcción. Polis. Revista Latinoamericana, 5.

Mahoney, J. (2003). The legacies of liberalism: Path dependence and political regimes in Central America. Johns Hopkins University Press. 
Matulis, B. S. (2013). The narrowing gap between vision and execution: Neoliberalization of PES in Costa Rica. Geoforum, 44, 253-260. https:// doi.org/10.1016/j.geoforum.2012.09.001

Meletis, Z., y Harrison, E. (2010). Tourists and turtles: Searching for a balance in Tortuguero, Costa Rica. Conservation and Society, 8(1), 26. https://doi.org/10.4103/0972-4923.62678

MINAE, SENARA, ICAA, MIDEPLAN, y IAEA. (2013). Agenda del Agua de Costa Rica. MINAE, SENARA, ICAA, MIDEPLAN e IAEA.

Molina, I. (1988). La alborada del capitalismo agrario en Costa Rica. Editorial UCR.

Molina, I., y Palmer, S. (2017). Historia de Costa Rica. Universidad de Costa Rica.

Monge, C. (2015). Desarrollo sostenible a la tica: Geopolítica y ambiente en la Administración Figueres Olsen (1994-1998). Revista Rupturas, 5(1), 1-21.

Montes Ruiz, A. P., y Durán Segura, L. (2019). Tres apuntes sobre la ciudad neoliberal en Costa Rica (1980-2017). Revista Arquis, 8(1), 1-23.

Ong, A. (2006). Neoliberalism as exception: Mutations in citizenship and sovereignty. Duke University Press.

PEN. (2018). Informe Estado de la Región 2018. Programa Estado de la Nación.

Ramírez, A. (2017). A political ecology of neoliberal multiculturalism: Social inclusion and market-based conservation in indigenous Costa Rica [Tesis de Doctorado]. Erasmus University Rotterdam.

Ribot, J. C., y Peluso, N. L. (2003). A theory of access. Rural Sociology, 69(2), 153-181.

Robbins, P. (2013). Political Ecology. Routledge.

Robinson, W. I. (2003). Transnational conflicts: Central America, social change and globalization. Verso.

Rovira, J. (1987). Costa Rica en los años ochenta. Editorial Porvenir.

Sader, S. A., y Joyce, A. T. (1988). Deforestation rates and trends in Costa Rica, 1940-1983. 11(1), 11-19.

Segura, F., y Moreira, J. M. (2013). La institucionalidad del sector agropecuario costarricense: Evolución y efectos sobre el desarrollo del sector [Informe de investigación]. Programa Estado de la Nación.

Seligson, M. (2001). ¿Problemas en el paraíso? La erosión en el apoyo al sistema político y la centroamericanización de Costa Rica. En J. Rovira 
Mas (Ed.), La democracia de Costa Rica ante el siglo XXI (pp. 87-120). University of Costa Rica Press.

Seligson, M. A. (2001). Costa Rican exceptionalism: Why the Ticos are different. En R. I. Camp (Ed.), Citizen views of democracy in Latin America (pp. 90-106). University of Pittsburgh Press.

Shiva, V. (1993). Monocultures of the mind: Perspectives on biodiversity and biotechnology. Zed Books.

Sojo, C. (1991). La utopía del estado mínimo. Coordinadora Regional de Investigaciones Económicas y Sociales.

Stott, P., y Sullivan, S. (2000). Political ecology: Science, myth and power. Arnold.

USAID. (1989). Costa Rica: Forest resources for a stable environment. USAID.

van Noorloos, F. (2011). Residential tourism causing land privatization and alienation: New pressures on Costa Rica's coasts. Development, 54(1), 85-90.

Vandermeer, J., y Perfecto, I. (2013). Breakfast of biodiversity: The political ecology of rain forest destruction. Food First.

Watts, M., y Peet, R. (2004). Liberating political ecology. Liberation ecologies: Environment, development, social movements, 2, 3-43.

Zárate Montero, D., y Ramírez García, R. (2016). Matriz energética de Costa Rica: Renovabilidad de las fuentes y reversibilidad de los usos de energía [Informe de investigación]. FES.

Zimmerer, K. S. (2011). "Conservation booms" with agricultural growth? Sustainability and shifting environmental governance in Latin America, 1985-2008 (Mexico, Costa Rica, Brazil, Peru, Bolivia). Latin American Research Review, 46, 82-114.

ALONSO RAMÍREZ COVER es costarricense. Doctor en Estudios de Desarrollo por el International Institute of Social Studies de la Erasmus University Rotterdam, Países Bajos. Docente de la Escuela de Ciencias Políticas e investigador del Centro de Investigación y Estudios Políticos de la Universidad de Costa Rica. 